\title{
Site testing Dome A, Antarctica
}

\author{
J.S. Lawrence ${ }^{\mathrm{a}^{*}}$, M.C.B. Ashley ${ }^{\mathrm{a}}$, M.G. Burton ${ }^{\mathrm{a}}$, X. Cui ${ }^{\mathrm{b}}$, J.R. Everett ${ }^{\mathrm{a}}$, B.T. Indermuehle ${ }^{\mathrm{a}}$, \\ S.L. Kenyon ${ }^{\mathrm{a}}$, D. Luong-Van ${ }^{\mathrm{a}}$, A.M. Moore ${ }^{\mathrm{c}}$, J.W.V. Storey ${ }^{\mathrm{a}}$, A. Tokovinin ${ }^{\mathrm{d}}$, T. Travouillon ${ }^{\mathrm{c}}$, \\ C. Pennypacker ${ }^{\mathrm{e}}$, L. Wang ${ }^{\mathrm{e}}$, D. York ${ }^{\mathrm{f}}$ \\ ${ }^{a}$ School of Physics, University of New South Wales, Australia \\ ${ }^{\mathrm{b}}$ Nanjing Institute of Astronomical Optics and Technology, China \\ ${ }^{\mathrm{c}}$ California Institute of Technology, USA \\ ${ }^{\mathrm{d}}$ Cerro-Tololo Inter-American Observatories, Chile \\ ${ }^{\mathrm{e}}$ Lawrence Berkeley Lab, University of California/Berkeley, USA \\ ${ }^{\mathrm{f}}$ University of Chicago, USA
}

\begin{abstract}
Recent data have shown that Dome C, on the Antarctic plateau, is an exceptional site for astronomy, with atmospheric conditions superior to those at any existing mid-latitude site. Dome $\mathrm{C}$, however, may not be the best site on the Antarctic plateau for every kind of astronomy. The highest point of the plateau is Dome A, some $800 \mathrm{~m}$ higher than Dome C. It should experience colder atmospheric temperatures, lower wind speeds, and a turbulent boundary layer that is confined closer to the ground. The Dome A site was first visited in January 2005 via an overland traverse, conducted by the Polar Research Institute of China. The PRIC plans to return to the site to establish a permanently manned station within the next decade. The University of New South Wales, in collaboration with a number of international institutions, is currently developing a remote automated site testing observatory for deployment to Dome A in the 2007/8 austral summer as part of the International Polar Year. This self-powered observatory will be equipped with a suite of site testing instruments measuring turbulence, optical and infrared sky background, and sub-millimetre transparency. We present here a discussion of the objectives of the site testing campaign and the planned configuration of the observatory.
\end{abstract}

Keywords: site testing, Antarctic astronomy

\section{INTRODUCTION}

It has long been recognised that the high, dry, cold sites on the Antarctic plateau should be ideal for astronomy (see Storey $^{1}$ for a recent review). Harper ${ }^{2}$ suggested that the low temperatures throughout the Antarctic plateau troposphere would result in a very low atmospheric thermal emission in the infrared. Gillingham ${ }^{3}$ predicted that the calm and stable atmosphere above the Antarctic plateau would result in weak turbulence, and that the absence of katabatic forced winds on the domes of the high plateau would result in only a very thin layer of surface turbulence.

These expectations motivated a series of site testing experiments at the US Amundsen-Scott South Pole station $\left(0^{\circ}\right.$ east, $90^{\circ}$ south, $2835 \mathrm{~m}$ elevation). Instruments demonstrated that the infrared sky emission was 1-2 orders of magnitude lower than typically found at mid-latitude sites ${ }^{4-7}$, and that the atmosphere held extremely low precipitable water vapour $^{8}$. The expectation of weak free-atmosphere turbulence was also confirmed. However, a very strong turbulent surface layer several hundred metres thick was found, resulting in only mediocre ground level seeing ${ }^{9-11}$.

The Italian/French Concordia station at Dome C (123 east, $75^{\circ}$ south, $3250 \mathrm{~m}$ elevation), lying at a higher altitude than South Pole, was expected to experience colder atmospheric temperatures, resulting in even lower thermal emission and lower water vapour content. Additionally, the local topography of Dome C indicated that the surface wind speeds should be lower, and thus the turbulent boundary layer should be confined closer to the surface ${ }^{2,12}$ These expectations were confirmed by a series of site testing experiments at this site. The sub-millimetre opacity and infrared atmospheric

*jl@phys.unsw.edu.au; phone 612 9385 5003; fax 61293856060 
emission was shown to be lower than observed at South Pole ${ }^{13,14}$, and while the wintertime surface boundary layer was found to exhibit relatively strong turbulence, this was confined to within $\sim 30 \mathrm{~m}$ of the surface ${ }^{15,16}$.

Dome A (74 east, $71^{\circ}$ south, $4100 \mathrm{~m}$ elevation) lies at the highest point of the Antarctic plateau. Regional meteorological climate models predict that the near-surface wind speeds at Dome A should be weaker than anywhere else on the plateau, as shown in Figure 1. These predictions have been confirmed by Automated Weather Station data from the site. Winds within the near-surface layer cause shear generation of turbulence; the near-ground turbulence at Dome A should thus be much lower than other high plateau sites. The higher altitude of Dome A is also expected to result in a significantly colder and drier atmosphere than other plateau sites ${ }^{17}$. We now plan to quantify these atmospheric characteristics at Dome A via the development of an autonomous self-powered observatory, PLATO (PLATeau Observatory), which contains a suite of site testing instrumentation. PLATO will be deployed to Dome A in the 2007/8 austral summer as part of the International Polar Year.

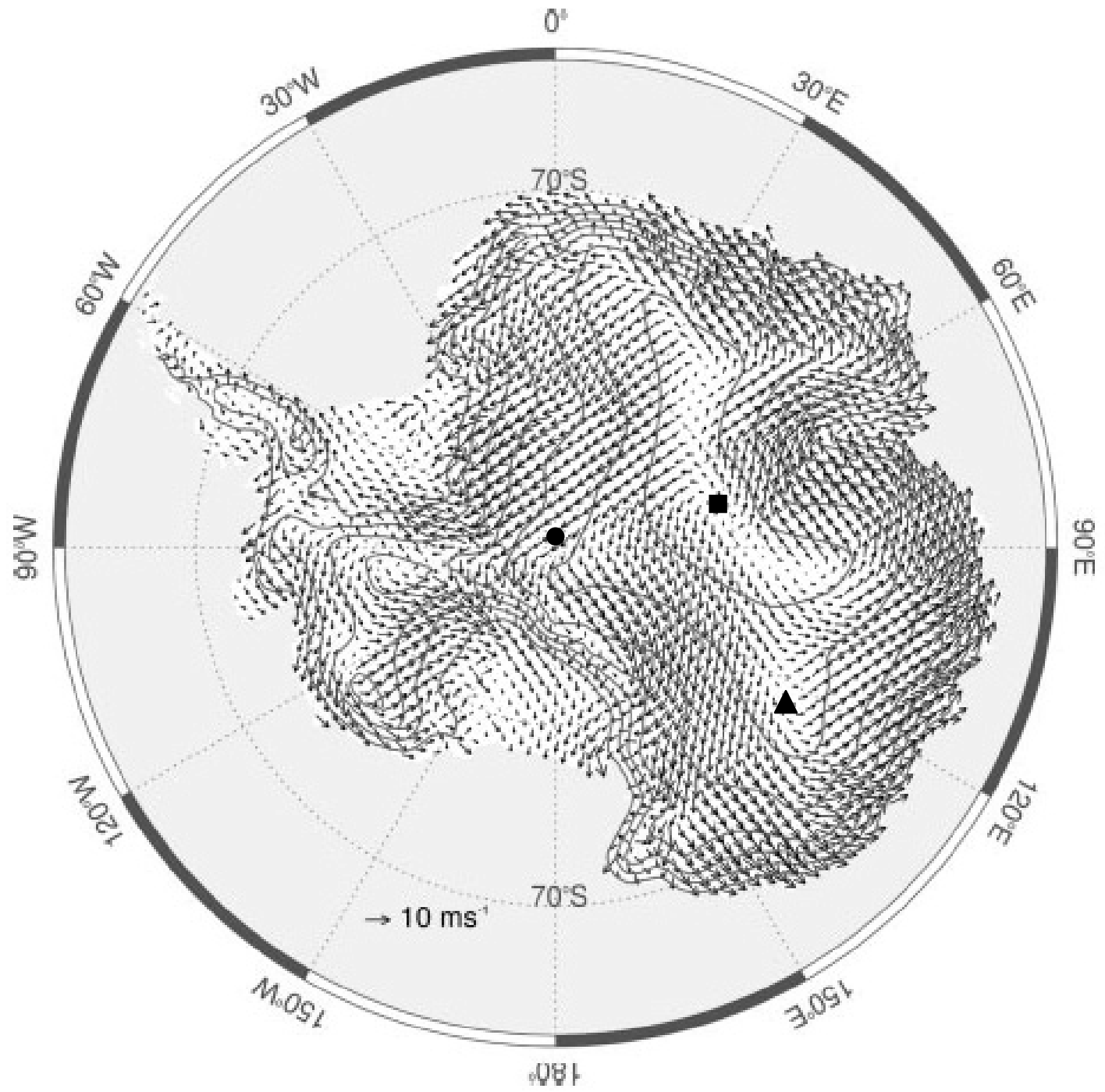

Figure 1. Mean winter (JJA) $7 \mathrm{~m}$ wind vector over Antarctica for 1980-93, from the high resolution Regional Atmospheric Climate Model (RACMO). Points marked are South Pole (circle), Dome C (triangle), and Dome A (square). Reproduced with permission from Van Lipzig et al. ${ }^{18}$. Copyright 2004 The Royal Meteorological Society. 


\section{ANTARCTIC SITE TESTING PROGRAMS}

South Pole station has been manned year round for the past fifty years, with power (from the station diesel generators), and communications (via several inclined almost-geostationary satellites) now available to any experiments located there. Early data from meteorological balloon launches and high frequency radiometers demonstrated the exceptional sub-millimetre opacity and stability, motivating the development of a number of sub-millimetre telescopes (e.g., Stark et al. ${ }^{19}$ ). The turbulent structure of the atmosphere was investigated during the 1995 winter via the launch of a series of microthermal balloon-borne probes ${ }^{10}$. Other site testing experiments at the South Pole have been conducted by the Automated Astrophysical Site Testing Observatory (AASTO) program ${ }^{20}$. Originally developed to demonstrate selfpowered operation, the AASTO, shown in Figure 2, was powered by a Thermo-Electric Generator, and linked to the local South Pole ethernet network for data transfer. Several years of testing demonstrated that the Thermo-Electric Generator power source was not sufficiently reliable, and thereafter the AASTO was powered from the South Pole station grid. The AASTO included instruments that measured the sub-millimetre atmospheric opacity, the integrated seeing down to ground level, the near- and mid-infrared sky brightness, and the near-surface turbulence from 30-900 m.
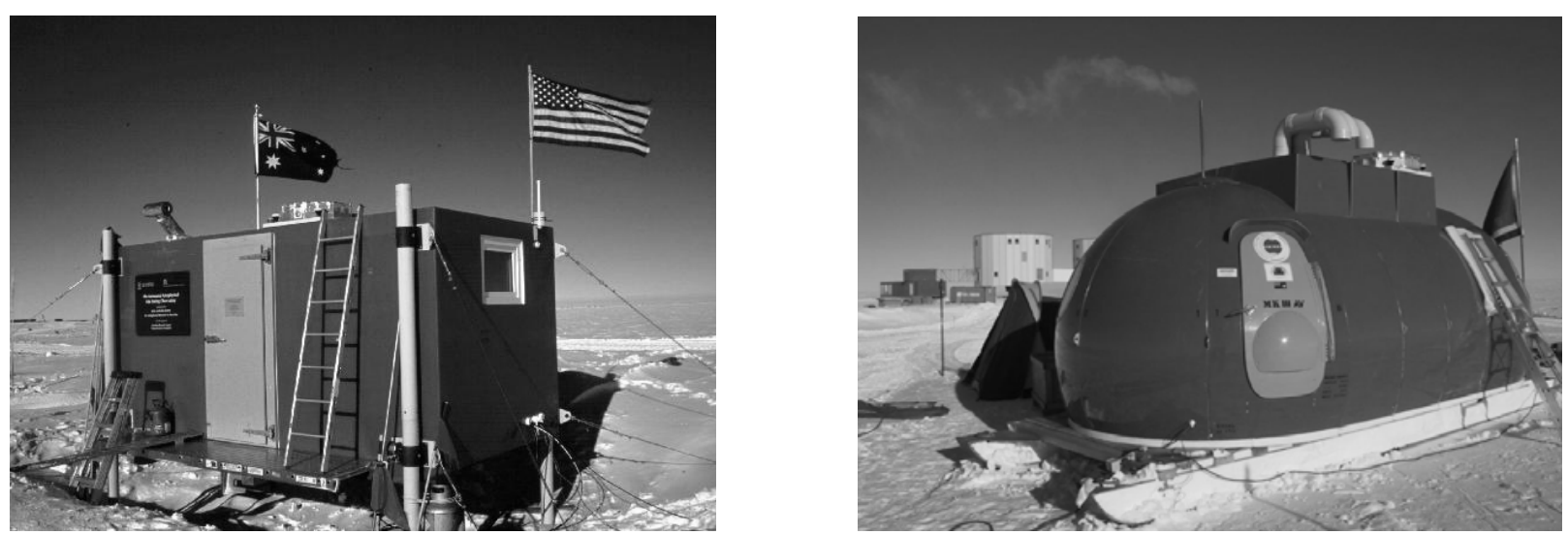

Figure 2: The AASTO at South Pole station (left) and the AASTINO at Dome C station (right).

Dome C has been manned during the summer months (Nov, Dec, Jan) since 1997, with year-round manned operation commencing with the completion of Concordia station in 2005. These logistic considerations have led to two different approaches to site testing at Dome C. The Concordiastro project, led by the University of Nice, has aimed to characterize the atmospheric turbulence at the site, with a series of instruments requiring on-site power and support. Instruments have included a number of Differential Image Motion Monitors (DIMMs) operated at various heights above ground level during the 2003-2005 summer seasons ${ }^{21}$ and year round since $2005^{16}$; a series of meteorological balloonborne probes launched during the 2000-2005 summer seasons ${ }^{22}$; and a series of microthermal balloon-borne probes launched during the first winter season of $2005^{16}$. The AASTINO (Automated Antarctic Site Testing INternational Observatory) campaign, led by the University of New South Wales, has focused on developing highly autonomous selfpowered instrumentation $^{23}$. The AASTINO, shown in Figure 2, was installed at Dome C in January 2005, and ran unattended during winter periods in 2003 and 2004. It was powered by a pair of Stirling engines running on Jet A1 aviation fuel and a pair of solar panels. Communication was via the Iridium satellite network. AASTINO instrumentation included a Multi-Aperture Scintillation Sensor (MASS) for the measurement of high-altitude turbulence, and the sub-millimetre radiometer (SUMMIT) and surface layer acoustics radar (SODAR) from the South Pole AASTO campaign.

The lack of an established base at the Dome A site leads to stringent requirements on an observatory dedicated to site testing. Only very basic technology low-power (few W) instruments have so far been deployed to the site, via the 2005 Polar Research Institute of China traverse. These included an Automated Weather Station developed by the Australian Antarctic Division, and an optical sky monitor developed by the University of New South Wales. The next generation of site testing observatory will require a high reliability power source of $1 \mathrm{~kW}$ or more to provide power to a range of 
sophisticated instrumentation measuring a variety of atmospheric parameters. It will require a robust command and control system which will be based on an upgrade of the AASTINO systems. The prohibitive cost and added complexity of a geostationary satellite ground station precludes the use of such a communications link. Communication will thus be via the Iridium satellite network, which although providing only a low bandwidth ( $<10000$ baud) has proven extremely reliable at Dome C. The PLATO structure will also be a modified version of the AASTINO, based on an external fiberglass casing with internal polyurethane insulation. The physical layout, currently under development, will ultimately be determined by the method of transportation-whether via overland traverse or Twin Otter aircraft. The PLATO system is likely to include a self erecting telescopic tower for instruments requiring elevations up to $\sim 30 \mathrm{~m}$.

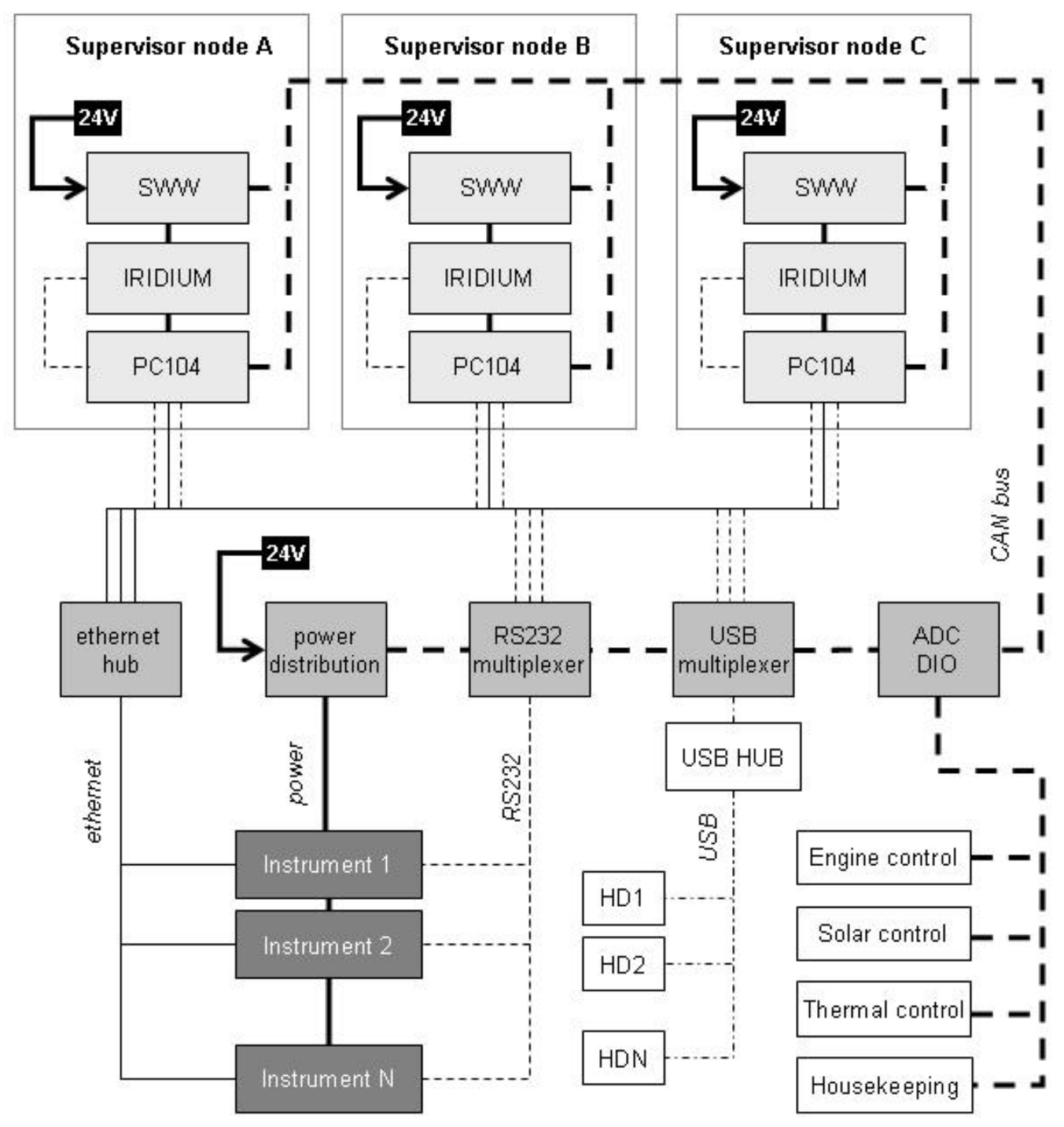

Figure 3: Schematic of the PLATO control and communications system. 


\section{PLATO POWER SUPPLY}

The power generation system for the PLATO is currently undergoing further development. At this stage is it proposed that primary power will be sourced from diesel engines, as these will use less fuel than the Stirling engine power system at Dome C. Similar to the AASTINO, secondary power for PLATO will be provided from a solar panel array with a maximum power-point tracker. An intelligent power distribution controller combines both sources to charge a primary and reserve 24V battery bank. The power distribution controller diverts any excess diesel power to a shunt regulator to prevent over-voltage conditions.

The diesel engine subsystem consists of a modular array of four to eight 0.35 litre single-cylinder engines. Each engine has its own intelligent controller that communicates with the control computer via a CAN (Control Area Network) bus. The engine controller can start and shut-down the engine via an electric starter. It controls the speed and monitors the engine health via temperature sensors and machine condition monitoring accelerometers. Only one engine is run at a time and the running sequence is determined via the engine health. When an engines health is low or it has had extended run time, a second engine is started before the original is shut down.

The diesel engines are run at constant speed and can deliver up to $1.5 \mathrm{~kW}$ of electrical power at $2000 \mathrm{RPM}$. The power output is determined by the amount of torque required. The low running speed is optimal for fuel consumption and wear, while the constant running speed allows for precise vibration control. Mechanical energy is converted to electricity via a high efficiency alternator with an internal switched-mode converter. Waste heat is recovered and is used to heat the PLATO, inactive diesel engines, and battery banks. Exhaust is scrubbed and waste heat is recovered before being released down wind. The valve timing and fuel delivery timing are tuned for the low atmospheric pressure. An environmentally controlled dynamometer is currently being built to allow for optimum tuning of these parameters before deployment.

Although ideally only one engine will ever be cold started, all engines require modification of external running ancillaries. All structural plastic components are replaced with metal substitutes that are thermal expansion compatible. The fuel is based on the readily available Jet A1 aviation fuel but has added cetane enhancers and lubricity modifiers to extend engine life. A large fuel filter bank ensures a clean fuel supply for a minimum of one year between servicing. Each engine's oil sump is also modified to allow for enough oil for one years continuous running and is filtered in its own large capacity filter bank.

During periods when there is sun available, between $1-2 \mathrm{~kW}$ of electrical power is generated from a solar panel array. The high reflectivity of the ice and low temperature allow for high gathering and conversion efficiency. During the summer months the complete observatory can be run entirely from solar power. During the spring/autumn months, solar power is used to reduce fuel consumption.

\section{PLATO COMMAND AND CONTROL}

A central supervisor computer system controls all aspects of the PLATO. A schematic of the control system is shown in Figure 3. The supervisor system is a fault tolerant modular network based on $250 \mathrm{k}$ baud CAN bus supervisor nodes. Each of the three supervisor nodes consists of a Super Wakey Wakey (SWW) watchdog timer, an Iridium L-band transceiver, and a PC104 computer stack. Each PC104 computer stack consists of a processor module (TPP3 $300 \mathrm{MHz}$ Mobile Pentium III with 512 MB SDRAM), power supply module (D104-PCS20), and multi-port module (COM-1270 Parvus serial, ethernet, CAN, DIO), with two 2 GB low temperature Flash Disc Hard Drives (FDHD) whose configuration can be swapped between master and slave.

The core elements of the supervisor nodes are the SWW boards. The PC104 sends a watchdog timer reset periodically on the CAN bus if it is operational (via internal self diagnostics). Each node is assigned a unique priority. If no watchdog reset above its priority is received, the SWW attempts to power up the PC104 module. If it fails to receive a watchdog signal, it power cycles the PC104 and exchanges master and slave flash drives to handle a drive failure. The system is designed such that two of the three computer nodes are powered up at any one time, one of which is the dominant or active unit, the other operates in standby mode. The SWW module is cold hardened and contains a thermistor to roughly estimate the temperature. If the temperature is too low, indicating power source failure, it will not allow a system to power up. All CAN bus transceivers are ESD protected and have internal logic-lock up prevention to 
avoid situations that could potentially lock the bus. Key areas of the PC104's FD hard drives are always mounted read only to prevent unexpected power loss from corrupting core operating system parameters. Volatile system directories are mounted in RAM drives to minimise flash memory wear, and are periodically backed up for logging purposes. Each supervisor is connected via RS232 to its own Iridium transceiver.

The CAN bus network contains an ADC/DIO bus board which communicates with vital PLATO systems. This board controls engine, solar power, and PLATO thermal management, and collects housekeeping data from a series of internal and external temperature and pressure monitors and web-cameras. Power to each of the site testing instruments is supplied via a central power distribution unit, consisting of a series of switched-mode power supplies, which is connected to the CAN bus network and controlled from the dominant supervisor computer. This unit also monitors instrument current loads.

In order to eliminate single-point failure modes, each of the instruments contains its own computer for instrument control and data storage. The PLATO supervisor computer hub communicates with each of these computers via a local ethernet network (using an ethernet multiplexer which assigns the gateway to the dominant supervisor hub). For redundancy (ethernet is not considered a high-reliability network) each instrument is also connected to the supervisor hub via an RS232 network (using a COM port multiplexer).

The low bandwidth of the Iridium communications link necessitates that the majority of data is stored on site and collected once per year during servicing. A key role of the supervisor control computers is thus to back up this data from each of the instruments. An array of USB hard drives is used for this mass storage. To account for the high altitude at Dome A, the USB mass storage resides inside either a pressurised or high density gas enclosure to ensure the correct hard drive head clearance. A USB multiplexer controlled via the CAN bus network is used to connect the USB array to the dominant supervisor computer. Each drive in the array is partitioned into a series of logical partitions that are mounted read-only except for the active writing partition. This minimises the chance of file system corruption destroying large blocks of data. Each day the active partition and drive set is rotated, maximising the chance of a good spread of data being received if file system corruption does occur.

\section{PLATO INSTRUMENT SUITE}

The PLATO system is designed to control and communicate with a range of instruments. Key design requirements for each of the instruments are that they are as simple as possible, with few moving parts. They must have their own robust and reliable computer and electronics systems and automated operation scripts. All components are low-temperature rated, and, in particular, mechanisms for the removal of snow and ice formation on any external windows must be employed. See Ashley et al. ${ }^{24}$ for a discussion of specific requirements for Antarctic instrumentation.

Key atmospheric properties to be measured by the PLATO include the structure of the atmospheric turbulence above the site (in particular it is essential to determine the height and magnitude of the surface boundary layer), the sky emission and transmission (at optical, infrared and sub-millimetre wavelengths), and the meteorological conditions (throughout the surface layer). Instruments for PLATO will be contributed by various collaborators as part of the International Polar Year “Astropoles” program. While the full complement of instruments is yet to be finalised, it is likely to comprise at least the following.

\subsection{Atmospheric Turbulence}

- $\quad$ MASS: The Multi-Aperture Scintillation Sensor measures high-altitude turbulence with low spatial resolution but high temporal resolution (see Kornilov et al. ${ }^{25}$ ). MASS instruments are typically mounted on a DIMM telescope. To obviate the complexity associated with a completely robotic winterised external pointing and tracking mount, the Dome C MASS instrument utilised a gimbal-fed refractive telescope system that was mounted inside the AASTINO ${ }^{26}$. PLATO will employ a modified version of this instrument.

- Sonics: a series of sonic anenometers measuring temperature, pressure and 3-dimensional wind speed vectors were installed on a $30 \mathrm{~m}$ tower at Dome C in early 2006. From these data the profiles of the boundary layer 
turbulence can be derived ${ }^{27}$. We envisage installing a similar set of sensors on a self-erecting tower as part of the PLATO. Their reliability in a low temperature environment, however, still needs to be determined.

- Lunar SHABAR: The lunar SHAdow Band And Ranging instrument employs an array of photodiodes which measure the variation of lunar intensity over time. From these scintillation statistics the profile of night-time turbulence within the ground layer can be derived ${ }^{28}$. A single array was installed at Dome C in early 2006. A modified version of this instrument, employing either a tracking mechanism or a series of independent arrays to increase the temporal coverage, is currently being designed for the PLATO.

- SODAR: A commercial acoustic radar (Remtech \#PA1) has been used successfully in Antarctic plateau conditions at South Pole and Dome $C^{11}$ for the measurement of turbulence in the near surface layer from 30$1000 \mathrm{~m}$. It is likely that this same instrument will be installed in the PLATO. The usefulness of this instrument is questionable, however, as we expect the turbulence above $30 \mathrm{~m}$ to be below the detection threshold of this instrument for the majority of time.

- $\quad$ Ground Layer SODAR: No commercial SODARs are capable of measuring turbulence within the ground layer below about $30 \mathrm{~m}$, as they are also measuring wind speed and direction and thus require a long integration time and volume. It is, however, possible for a SODAR that only measures turbulence to sample the lowest $50 \mathrm{~m}$ with a reasonable $(\sim 1 \mathrm{~m})$ resolution. We are currently investigating the design of this instrument for PLATO.

\subsection{Atmospheric Emission and Transmission}

- $\quad$ SBC: As part of the GATTINI project an optical Sky Background Camera was installed at Dome C in 2006. This camera employs a commercial USB CCD array with an f/2.8 lens giving a $\sim 4$ degree FOV. It measures the magnitude of the optical sky flux in the SDSS g' band, and monitors the temporal variation of stars close to the celestial South Pole ${ }^{29}$. An upgraded version of this instrument with a series of optical filters will be installed in the PLATO.

- $\quad$ Nigel: The Nigel instrument consists of a fibre-fed optical spectrometer designed to measure the optical sky spectrum at various elevation angles ${ }^{30}$. This instrument aims to determine the magnitude and spatial and temporal distribution of aurora above Dome A. As Dome A lies within the auroral oval, aurora is likely to contribute significantly to the optical sky brightness ${ }^{31}$.

- IRMA/McSHMISM: The Infrared Radiometer for Millimetre Astronomy developed by the University of Lethbridge employs a steerable single element HgCdTe detector operating at 20 micron to determine the atmospheric emission at this wavelength $^{32}$. The McSHMISM, currently under development, employs a stationary infrared array that is coupled to an infrared spectrometer. In this instrument wavelength is dispersed across one axis of the detector, and a $90^{\circ}$ swarth of the sky from zenith to horizon is imaged across the other axis. It is likely that only one of these two instruments will be deployed with the PLATO.

- $\quad$ SUMMIT/DAFTS: The SUMMIT is a sub-millimetre radiometer, which uses a pyroelectric detector in skydip mode to measure the opacity in the 350 or $200 \mu \mathrm{m}$ atmospheric windows. It has proven reliable for operation in Antarctic plateau conditions at South Pole and Dome $C^{13}$. The Dome A Fourier Transform Spectrometer is an instrument currently under development that will employ a superconducting transition-edge bolometer to obtain atmospheric emission and transmission spectra for a wide region of sub-millimetre wavelengths. It is likely that only one of these two instruments will be deployed with the PLATO.

\subsection{Meteorological Conditions}

- $\quad$ AWS: standard Automatic Weather Station sensors measuring pressure, temperature, humidity, wind speed and wind direction will be installed external to the PLATO at various heights (possibly tower mounted).

- $\quad$ ASC: The PLATO will be equipped with an All-Sky Camera to measure cloud cover statistics and auroral distribution. This will likely consist of a wide-field integrating optical CCD array or video camera. Another 
possibility being considered is the use of a mid-infrared micro-bolometer array so that daytime cloud cover can also be evaluated.

- Visibility: a commercial visibility meter (such as the Vaisala \#FD12) measuring precipitation rates of snowfall and snow accumulation rates will be installed external to the PLATO.

\section{CONCLUSION}

While Dome A is potentially the best ground-based astronomical site, it is also the most remote and coldest location on the planet. The development of an automated site testing observatory for this site is thus a difficult challenge. The observatory must be entirely self-powered with a reliable multiply redundant hybrid power generation system. The observatory must contain sufficient instruments to obtain the wide variety of atmospheric data required. Each instrument must operate consistently and autonomously in the extreme external conditions. The command and communications system for the observatory must control all aspects of power generation, instrumentation and data storage, and must be robust against the many potential failure points. The PLATO laboratory described in this paper is currently being designed based on these principles. We plan to deploy the PLATO to Dome A during the 2007/8 Antarctic summer season. Previous experiences in the operation of self-powered observatories at the South Pole (AASTO) and Dome C (AASTINO), and in the design of many unique Antarctic site testing instruments, will prove invaluable in this design process.

\section{REFERENCES}

1. J.W.V. Storey, “Astronomy from Antarctica”, Antarctic Science, 17, 555, 2005.

2. D.A. Harper, "Infrared astronomy in Antarctica", in Astrophysics in Antarctica, Amer. Inst. Phys. Conf. Proc. 198, 123, 1989.

3. P.R. Gillingham, "Prospects for an Antarctic Observatory", Proc. ASA, 9, 55, 1991.

4. M.C.B. Ashley, M.G. Burton, J.W.V. Storey, J.P. Lloyd, J. Bally, J.W. Briggs, D.A. Harper, "South Pole observations of the near-infrared sky brightness”, PASP, 108, 721, 1996.

5. H.T. Nguyen, B.J. Rauscher, D.A. Harper, R.F. Loewenstein, R.J. Pernic, S.A. Severson, M. Hereld, "The South Pole Near Infrared Sky Brightness”, PASP, 109, 718, 1996.

6. A. Phillips, M.G. Burton, M.C.B. Ashley, J.W.V. Storey, J.P. Lloyd, D.A. Harper, J. Bally, “The near-infrared sky emission at the South Pole in winter”, ApJ, 527, 1009, 1999.

7. M.A. Chamberlain, M.C.B. Ashley, M.G. Burton, A. Phillips, J.W.V. Storey, D.A. Harper, "Mid-infrared observing conditions at the South Pole", ApJ, 535, 501, 2000.

8. R.A. Chamberlin, "South Pole submillimeter sky opacity and correlations with radiosonde observations", $J$. Geophys. Res., 106, 20101, 2001.

9. R.F. Loewenstein, C. Bero, J.P. Lloyd, F. Mrozek, J. Bally, Bally, D. Theil, “Astronomical Seeing at the South Pole”, ASP Conf. Ser., 141, 296, 1998.

10. R. Marks, J. Vernin, M. Azouit, J.F. Manigault, C. Clevelin, "Measurements of optical seeing on the high Antarctic plateau”, A\&ASS, 134, 161, 1999.

11. T. Travouillon, M.C.B. Ashley, M.G. Burton, J.W.V. Storey, R.F. Loewenstein, "Atmospheric turbulence at the South Pole and its implications for astronomy”, A\&A, 400, 1163, 2003.

12. R. Marks, "Astronomical seeing from the summits of the Antarctic plateau", A\&A, 385, 328, 2002.

13. P.G. Calisse, M.C.B. Ashley, M.G. Burton, M.A. Phillips, J.W.V. Storey, S.J.E. Radford, J.B. Peterson, "Submillimeter site testing at Dome C, Antarctica", PASA, 21, 256, 2004.

14. V.P. Walden, M.S. Town, B. Halter, J.W.V. Storey, "First measurements of the infrared sky brightness at Dome C, Antarctica”, PASP, 117, 300, 2005.

15. J.S. Lawrence, M.C.B. Ashley, A.A Tokovinin, T. Travouillon, “ Exceptional astronomical seeing conditions above Dome C in Antarctica”, Nature, 431, 278, 2004.

16. K. Agabi, E. Aristidi, M. Azouit, E. Fossat, F. Martin, T. Sadibekova, J. Vernin, A. Ziad, "First whole atmosphere nighttime seeing measurements at Dome C, Antarctica”, PASP, 118, 344, 2006. 
17. J.S. Lawrence, "Infrared and sub-millimetre atmospheric characteristics of Antarctic high plateau sites”, $P A S P, \mathbf{1 1 6}$, 482, 2004.

18. N.P.M. van Lipzig, J. Turner, S.R. Colwell, M.R. van Der Broeke, “The near-surface wind field over the Antarctic continent”, Int. J. Climatology, 24, 1973, 2004.

19. A.A. Stark, J. Bally, S.P. Balm et al. (23 additional authors), "The Antarctic Submillimeter Telescope and Remote Observatory (AST/RO)”, PASP, 113, 567, 2001.

20. J.W.V. Storey, M.C.B. Ashley, M.G. Burton, “An automated astrophysical observatory for Antarctica”, PASA, 13, 34, 1996.

21. E. Aristidi, A. Agabi, E. Fossat, M. Azouit, F. Martin, T. Sadibekova, T. Travouillon, J. Vernin, A. Ziad, "Site testing in summer at Dome C, Antarctica ”, A\&A, 444, 651, 2005.

22. E. Aristidi, A. Agabi, M. Azouit, E. Fossat, J. Vernin, T. Travouillon, J.S. Lawrence, C. Meyer, J.W.V. Storey, B. Halter, W.L. Roth, V. Walden, “An analysis of temperatures and wind speeds above Dome C, Antarctica”, A\&A, 430, 739, 2005.

23. J.S. Lawrence, M.C.B. Ashley, J.W.V. Storey, “A remote, autonomous laboratory for Antarctica with hybrid power generation”, Aust. J. Elect. Electron. Eng., 2, 1, 2005.

24. M.C.B. Ashley, M.G. Burton, J.S. Lawrence, J.W.V. Storey, "Robotic telescopes on the Antarctic plateau”, Astron Nach., 325, 619, 2004.

25. V. Kornilov, A. Tokovinin, O. Vozyakova, A. Zaitsev, N. Shatsky, S.F. Potanin, M.S. Sarazin, "MASS: a monitor of the vertical turbulence distribution”, Proc. SPIE, 4839, 837, 2003.

26. J.S. Lawrence, M.C.B. Ashley, J.P. Lloyd, A. Tokovinin, M. Swain, S. Kenyon, J.W.V. Storey, “A robotic instrument for measuring high altitude atmospheric turbulence from Dome C, Antarctica ”, Proc. SPIE, 5489, 174, 2004.

27. T.W. Horst, S.P. Oncley, S.E. Larsen, M. Nielsen, and C.W. Fairall, “Corrections to Power Spectra Measured by CSAT3 and Solent Sonic Anemometers”, Proceedings from EGU General Assembly, Austria, April, 2005.

28. P. Hickson, K. Lanzetta, "Measuring Atmospheric Turbulence with a Lunar Scintillometer Array", PASP, 116, 1143, 2004.

29. A.M. Moore, E. Aristidi, M.C.B. Ashley, M.Candidi, J.R. Everett, S.L. Kenyon, J.S. Lawrence, A.Phillips, B. Le Roux, R. Ragazzoni, P.Salinari, J.W.V. Storey, M.Taylor, T. Travouillon, "The Gattini cameras for optical skybrightness measurements in Antarctica”, Proc. SPIE, 6269, in press, 2006.

30. S.L. Kenyon, M.C.B. Ashley, J.R. Everett, J.S. Lawrence, J.W.V. Storey, "Nigel and the optical sky brightness at Dome C, Antarctica”, Proc. SPIE, 6267, this proceedings, 2006.

31. J.T. Dempsey, J.W.V. Storey, M.A. Phillips, “Auroral contribution to sky brightness for optical astronomy on the Antarctic plateau", PASA, 22, 91, 2005.

32. R.R. Phillips, D.A. Naylor, J. diFrancesco, B. Gom, "Initial results of field testing an infrared water vapor monitor for millimeter astronomy (IRMA III) on Mauna Kea”, Proc. SPIE, 4589, 146, 2004. 